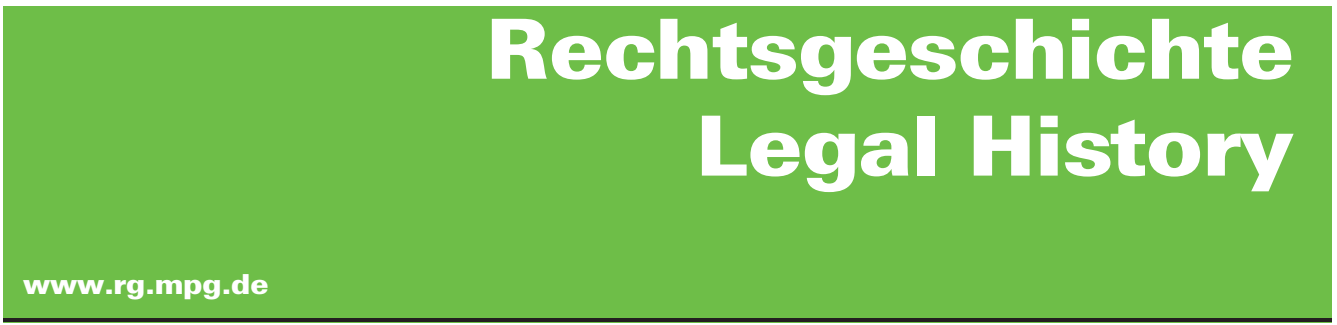

http://www.rg-rechtsgeschichte.de/rg20

$\mathrm{Rg} 202012 \quad 356-357$

Zitiervorschlag: Rechtsgeschichte - Legal History Rg 20 (2012)

http://dx.doi.org/10.12946/rg20/356-357

\title{
Claudio Ferlan
}

\section{Oggetti in viaggio}

Le missioni gesuitiche nelle Indie d'occidente (sec. XVI) 


\section{Claudio Ferlan}

\section{Oggetti in viaggio}

Le missioni gesuitiche nelle Indie d'occidente (sec. XVI)

Con la bolla Licet debitum (18 ottobre 1549) papa Paolo III concesse alcune facoltà straordinarie alla Compagnia di Gesù, riconoscendone la dimensione missionaria, così come era stato fatto in precedenza per altri ordini. Ai gesuiti veniva cos̀̀ esplicitamente consentito di dimorare nelle terre degli «infedeli», di comunicare con loro e di esercitare tutta una serie di funzioni canoniche nei luoghi dove la Santa Sede non sarebbe potuta arrivare. Qualche mese prima era iniziata la missione gesuitica nelle Indie occidentali, con l'arrivo di Manuel de Nóbrega in Brasile, dando il via così a una continua espansione dell'ordine nel Nuovo Mondo, caratterizzata da un'attenzione molto marcata alla comunicazione e alla trasmissione del sapere.

Attraverso esempi tratti dalla storia della Compagnia, propongo una riflessione sul contributo di alcuni oggetti alla costruzione di uno spazio normativo atlantico, giuridico e morale; ritengo infatti che certe tipologie di cose materiali si siano rivelate fondamentali per definizione e sviluppo di ordini normativi propri dell'esperienza ibero-americana (diritto canonico, teologia morale e liturgia, come esplicitato nell'invito al dibattito). Con particolare riferimento a quanto era importato dall'Europa in America mi chiedo: quale significato assumono determinati strumenti di evangelizzazione prima, durante e dopo la navigazione? In tal senso, lo spazio atlantico va considerato tenendo conto non solo dei luoghi di partenza e approdo, ma anche del percorso.

Riferendomi alla Compagnia di Gesù, voglio richiamare il fondamentale contributo che le fonti esterne all'ordine possono dare alla ricostruzione della sua storia: primi tra tutti i documenti pontifici, capaci di mettere in luce relazioni e problematiche proprie della spesso complessa dialettica tra gesuiti, Santa Sede, autorità religiose e secolari.

Gli spazi geografici di riferimento sono i tre nuclei fondamentali per l'evangelizzazione cinquecentesca di quella che oggi conosciamo come America Latina: Brasile, Perù e Messico. La scelta di mantenere uno spettro molto ampio è dettata dall'interesse a promuovere un dibattito anche in chiave comparativa.
Parto da un esempio preso dagli esordi della presenza gesuitica nel Nuovo Mondo. Il 9 agosto 1549 padre Nóbrega scrive al proprio superiore a Lisbona, Simão Rodrigues, sottolineando la necessità di vestiti femminili, così da poter coprire le donne convertite «per l'onestà della religione cristiana». Le donne brasiliane, infatti, sono solite «stare nude in mezzo ai cristiani nella chiesa», disturbando così l'apprendimento dei neofiti mentre i padri pregano e catechizzano. Una richiesta simile cosa ci rivela della possibilità di dialogo $\mathrm{e}$ comprensione tra culture? Possono bastare i vestiti per modificare i costumi e introdurre nuove norme etiche e comportamentali?

Prendiamo ora in considerazione l'oggetto che più di ogni altro esemplifica la comunicazione del sapere: il libro. Nella stessa lettera, Nóbrega chiede libri, sottolineandone la gran necessità in ragione dei dubbi frequenti che vengono sollevati in quei luoghi, dubbi che è incessantemente chiamato a risolvere in prima persona. Da una seconda lettera di Nóbrega a Rodrigues (6 gennaio 1550) sappiamo che sono arrivate due casse di libri e dalla successiva corrispondenza allarghiamo le nostre conoscenze: si tratta soprattutto di testi scolastici e di dottrina, acquistati e spediti grazie alla munificenza di corte portoghese e Santa Sede. Come vengono proposti alle popolazioni indigene? Come vengono letti e interpretati?

Ben informato dell'esperienza dei confratelli nelle Indie orientali e occidentali, al momento di partire, padre Jerónimo Ruiz de Portillo - capo della prima spedizione gesuitica verso il Perù scrive al generale Borja (2 gennaio 1568) di aver ricevuto dal re di Spagna il denaro necessario all'acquisto di numerosi libri, destinati ad essere utilizzati nel collegio da aprirsi a Lima e per la formazione dei gesuiti già presenti in loco. Una lettera di Juan de la Plaza per il generale Mercuriano (3 giugno 1574) racconta il naufragio di una nave destinata ad accompagnare alcuni padri gesuiti in Messico e in Perù; l'inconveniente ha causato la perdita di Costituzioni della Compagnia di Gesù, regole, uffici, biografie di Ignazio. Si tratta, come è ben evidente, di testi normativi, utili e necessari al trasferimento culturale delle regole di 
vita e condotta della Compagnia di Gesù dall'una all'altra parte dell'Atlantico. Di questi Plaza chiede un nuovo invio, aggiungendo all'elenco le edizioni delle summae delle Congregazioni Generali dell'ordine. Come far fronte alle mancate spedizioni, ai naufragi, alle lunghe attese necessarie prima di poter utilizzare i libri richiesti? Una risposta, ma non l'unica, si trova nella costruzione di stamperie nei luoghi di missione, un campo di investigazione di sicura rilevanza.

Il testo scritto è spesso utilizzato nel corso del viaggio di missione per trasmettere regole di comportamento ai marinai che lavorano nelle navi sulle quali sono imbarcati i missionari. Spesso identificato con il peccatore, il marinaio assume nell'immaginario trasmesso dalle fonti gesuitiche le caratteristiche, proprie o immaginate, dell'indigeno, primo destinatario dell'evangelizzazione.

In conclusione, intendo soffermarmi su un'altra presenza costante nel bagaglio dei gesuiti: le reliquie. Richieste dal Brasile, dal Messico e dal Perù, esse sono necessarie alla consacrazione delle chiese ma si rivelano anche importanti strumenti di predicazione.

In una lettera scritta dal Brasile al generale Laynez, il gesuita José de Anchieta (1 giugno 1560) racconta dell'imposizione di reliquie «de los Sanctos» al collo delle partorienti per accompagnarle al buon parto. Si tratta di una pratica tipica della devozione europea, per la quale nel secolo successivo saranno utilizzate con frequenza le immagini di Ignazio e Francesco Saverio. Quali forme assume la trasposizione di questa fede nelle reliquie? L'importazione di regole di comportamento identiche a quelle europee rischia di trovare un'interpretazione diversa nel luogo di missione? Quello che ad est dell'Atlantico è devozione rischia di essere scambiato, a ovest, per idolatria?
A proposito dell'utilizzo degli oggetti sacri durante il viaggio, interessante è il racconto di padre Rui Pereira in una lettera inviata ai propri confratelli in Portogallo (6 aprile 1561): nella navigazione tra Bahia e Pernambuco, assaliti da un fortunale, lui e i confratelli si salvano grazie all'aiuto di Agnus Dei e reliquie gettate in mare. Otto anni dopo (marzo 1569), una nave che trasporta due gesuiti membri della seconda spedizione in Perù viene assalita da una tremenda tempesta in mare aperto. A salvarli quando ormai - secondo i loro racconti - stanno per inabissarsi, è un piccolo pezzo della croce di Cristo, affidato da Pio V al gesuita Juan Casasola: immerso in mare e legato al timone, esso consente all'imbarcazione di riprendere la rotta. La reliquia sarà poi recuperata $\mathrm{e}$ si convertirà in oggetto di devozione e strumento di predicazione in terra peruviana: il valore acquisito durante il viaggio trasforma la reliquia in qualcosa di nuovo, in uno strumento divino dal potere miracoloso. Anche in questo caso, si pongono questioni simili a quelle proposte sopra, relative alla difficile definizione della fede nelle immagini e negli oggetti di cui si occuperà con grande sforzo il testo del catechismo prodotto all'interno del Terzo concilio di Lima. Quali esempi abbiamo di oggetti che si trasformano grazie al viaggio e che vivono una vita nuova determinata dalla reinterpretazione del loro valore e significato una volta «approdati» in America?

Fonti: America Pontificia I, 606-608; Monumenta Brasiliae SJ I, 131, 168; III, 254, 326; Monumenta Peruana SJ I, 164, 602-606, 689-691; Monumenta Mexicana SJ I, 104, 107-108, 213. 